\title{
A RELAÇÃO ENTRE CAPACIDADE ABSORTIVA, DINAMISMO AMBIENTAL E DESEMPENHO NO SETOR VINÍCOLA DO SUL DO BRASIL
}

Carlos Ricardo Rossetto ${ }^{1}$ José Pedro Silveira Fuchs ${ }^{1}$ Carlos Eduardo Carvalho ${ }^{2}$ Jorge Oneide Sausen ${ }^{3}$

\footnotetext{
${ }^{1}$ Universidade do Vale do Itajaí

${ }^{2}$ Universidade do Oeste de Santa Catarina (UNOESC)

${ }^{3}$ Universidade Regional do Noroeste do Estado do Rio Grande do Sul
} 


\section{A RELAÇÃO ENTRE CAPACIDADE ABSORTIVA, DINAMISMO AMBIENTAL E DESEMPENHO NO SETOR VINÍCOLA DO SUL DO BRASIL}

\section{Resumo:}

Este estudo procura investigar a relação entre a capacidade absortiva, o dinamismo ambiental e o desempenho da firma utilizando como universo amostral o setor vinícola do Sul do Brasil. Foram analisados 77 questionários válidos em uma pesquisa com produtores de uvas finas dos Estados do RS e SC, sendo aplicado o software SmartPLS para avaliar os relacionamentos propostos nas hipóteses. Como resultado, concluiu-se que é positivo e significante o efeito da Capacidade Absortiva no Desempenho, positivo também o efeito do Dinamismo Ambiental na Capacidade Absortivada e não foi suportada a hipótese da influência do dinamismo ambiental no desempenho nas empresas vinicolas pesquisadas. As conclusões se mostraram importantes como contribuição teórica e prática sobre a relevância da capacidade de absorção de conhecimento e o seu relacionamento com o Dinamismo Ambiental e o Desempenho.

Palavras-Chave: Capacidade Absortiva, Dinamismo Ambiental, Desempenho, Setor Vitivinícola.

\section{Introdução}

Diante da crescente competitividade e abertura de mercados, Li e LIU (2012) afirmam que é virtualmente impossível à manutenção de vantagem competitiva permanente, havendo a necessidade de mudança da visão das organizações para vantagens competitivas temporárias e que se exaurem rapidamente.

O dinamismo e a complexidade do ambiente da firma vão estabelecer padrões de reação que, por sua vez, vão exigir capacidades com nível de sofisticação e grau de adaptabilidade variável para uma resposta eficaz à incerteza ambiental. Tal dinamismo, se por um lado apresenta ameaças aos objetivos da firma, provocando mudanças rápidas no ambiente de negócios, por outro oferece oportunidades que poderão ser aproveitadas em função da utilização do conhecimento acumulado para atualizar os mecanismos usados no enfrentamento das novas realidades do mercado (HSIEN et al., 2009)

Este posicionamento das organizações em relação ao ambiente externo vai ser influenciado, preponderantemente, pela quantidade de informações que a firma consegue obter deste mesmo ambiente. Esta carga excessiva de dados é fruto das características do ambiente, gerando dinamismo, incerteza e provocando impacto nas decisões que devem ser tomadas. Para que as organizações sobrevivam neste ambiente, deverão desenvolver capacidades de processar as informações para tomadas de decisões mais eficazes (TEIXEIRA; ROSSETTO; CARVALHO, 2009).

Lane et al (2006) ressalta que a capacidade absortiva é um dos mais importantes construtos a emergir na pesquisa organizacional nas décadas recentes, para responder as dinâmicas do ambiente. Camisón e Forés (2010) sustentam a ampliação dos estudos sobre capacidade absortiva dada a importância que a busca de informações externas na área do conhecimento tem para as organizações.
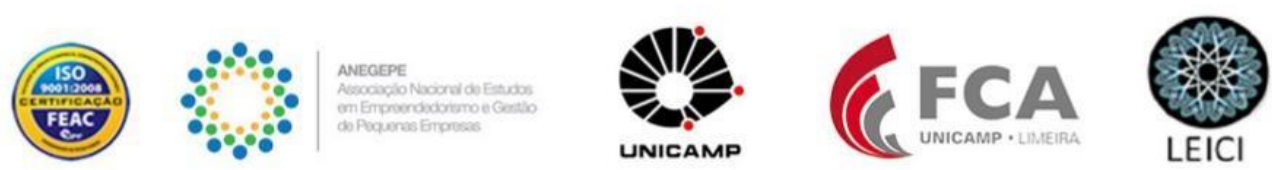
Para consolidar este cenário, o estudo de Li e Liu (2011) ressalta estas lacunas científicas, afirmando que a maioria dos estudos sobre capacidades absortivas provêm de mercados desenvolvidos com normas reguladoras bem estabelecidas, sabendo-se pouco das mesmas em mercados de economias em transição, como é o caso do Brasil, o que reforça a atualidade deste estudo e suas conclusões. Além disso, surge a oportunidade de contribuir teoricamente, estendendo a investigação ao universo das micro e pequenas empresas, área de pesquisa importante por sua contribuição na composição da economia brasileira, mas pouco abordada nos trabalhos sobre capacidade absortiva (LI; LIU, 2011).

No trabalho de Ndiege et al. (2012) sobre a relevância da capacidade absortiva para pequenas e grandes empresas, os autores salientam a carência de estudos neste campo em países em desenvolvimento, e reforçam a possibilidade de comparação destas pesquisas com as de países desenvolvidos para uma compreensão global do construto, inclusive com relação à sua influência no desempenho das pequenas e médias empresas.

Devido à sua importância no contexto organizacional, há necessidade de mais pesquisas que analisem detalhadamente o construto, evidenciando suas dimensões e o papel na absorção de conhecimento externo, pela formação da base informacional tácita e explícita da firma, além de sua influência no desempenho e na construção de vantagem competitiva no ambiente.

Portanto, a partir da justificativa do por que realizar a pesquisa e da contribuição teórica e empírica que os resultados trarão, o objetivo do artigo é verificar as relações existentes entre a capacidade absortiva, dinamismo ambiental e desempenho nas Pequenas e Médias Empresas da indústria do vinho nos Estados de Santa Catarina e Rio Grande do Sul.

\section{Capacidade Absortiva}

Em seu trabalho seminal sobre o tema, Cohen e Levinthal $(1989,1990)$ abordam a importância do P\&D como geradores de informação para a organização inovar e sugerem que, além deste processo de geração de novos conhecimentos, eles potencializam a habilidade da firma de assimilar e implementar a informação existente. Esta habilidade faz com que a empresa acumule, ao longo do tempo, uma relevante base de conhecimento. Eles chamaram este processo de "capacidade de aprendizado" ou "capacidade absortiva" da organização, numa primeira definição do que, mais tarde, se consolidou simplesmente como "capacidade absortiva" (COHEN; LEVINTHAL, 1989).

Durante a década passada, alguns autores apresentaram uma reconceitualização da capacidade absortiva, sendo a principal delas feita por Zahra e George (2002), que a dividiram em quatro dimensões: Aquisição, Assimilação, Transformação e Aplicação. Esta subdivisão tornou mais operacional à mensuração da capacidade absortiva de uma firma mesmo porque, conforme Levin et al. (2011), há uma série de metarotinas internas e externas constituindo o construto de capacidade absortiva. E é este modelo que será utilizado para mensurá-la neste estudo.

Segundo estes autores, a primeira dimensão, Aquisição, é formada pela identificação e aquisição de informações externas novas e relevantes para as operações da firma. Conhecimento anterior influencia fortemente esta dimensão. A segunda dimensão, Assimilação, representa a inclusão, conversão e interpretação da informação adquirida. Durante a terceira dimensão,
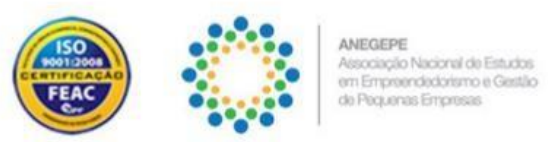

Realizadores:
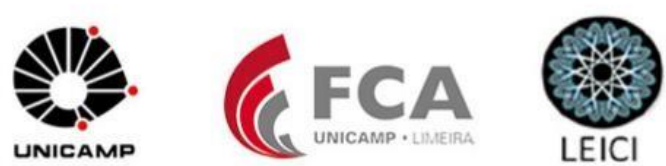
Transformação, o conhecimento novo e o existente são combinados, através do fomento de rotinas que facilitam esta internalização. Na quarta dimensão, Aplicação, são incluídas as rotinas que propiciam à firma a incorporação destes novos conhecimentos nas suas operações. Esta divisão em várias dimensões (com suas respectivas rotinas) facilitou o desenvolvimento de instrumentos de medida para uma avaliação mais consistente da capacidade absortiva.

\section{Dinamismo Ambiental}

Segundo Li e Liu (2012), o dinamismo é interpretado como impredicabilidade, isto é, o grau de mudança e inovação em uma determinada indústria, assim como a incerteza ou impossibilidade de previsão comportamental por parte dos consumidores e concorrentes.

Quando o dinamismo ambiental aumenta, com rápidas alterações em tecnologia, preferências dos consumidores, recursos cada vez mais disputados e aumento da competição, decisões estratégicas rápidas precisam ser tomadas pelos gerentes, ao mesmo tempo em que ajustes e estratégias inovadoras devem ser implementadas (D’AVENI, 1994). Quanto mais dinâmico é o ambiente, mais pertinente se torna a adoção de estratégias de inovação para enfrentar a concorrência, o que caracteriza de certa forma um comportamento determinista. $\mathrm{O}$ grau de dinamismo ambiental é considerado como fator de restrição a determinados fluxos de financiamento e captação de recursos, que se mostram avessos ao risco e à instabilidade inerentes à turbulência do ambiente dinâmico (ANDERSEN, 2005).

Segundo D'Aveni (2010), em ambientes supercompetitivos os recursos são difíceis de obtenção e, portanto, percepção eficiente, ajustes e implantação de mudanças no momento certo e de acordo com as variantes ambientais, são maneiras das organizações conseguirem vantagens competitivas de curto tempo. O mesmo autor afirma que no caso específico das micro, pequenas e médias empresas, o dinamismo ambiental representa papel decisivo no comportamento dos que decidem, em parte devido à pequena ou nenhuma capacidade que estas organizações têm de influenciar o seu ambiente, sofrendo diretamente a ação das mudanças e da incerteza ambiental percebida. A maior parte dos estudos que analisam dinamismo ambiental é ancorada no universo de médias e grandes empresas, com uma quantidade limitada de estudos em profundidade que incluam em sua maioria pequenas empresas (MILES; COVIN; HEELY, 2015).

A existência de fatores externos do ambiente é normalmente citada como tendo grande influência na viabilidade e no crescimento de pequenas e médias empresas, além de normas regulatórias governamentais e, mais recentemente, ambientais (BOSKIN, 1984). Além disso, o dinamismo ambiental é percebido de forma diferente pelas empresas, especialmente as pequenas e médias. Algumas percebem o ambiente com reduzido grau de incerteza, enquanto que, para outras, o ambiente é de elevada percepção de incerteza (TEIXEIRA; ROSSETTO; CARVALHO, 2009).

Em se tratando do objeto de estudo, o setor vitivinícola, de acordo com Farias (2014), é um excelente exemplo de adaptação e reação ao dinamismo do ambiente, pois, além das iniciativas individuais das firmas para adaptação a novas situações de mercado, percebe-se a cooperação entre os agentes econômicos na forma de associações e estabelecimento de vínculos
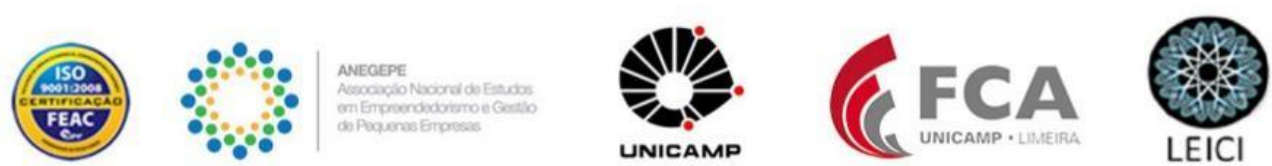
cooperativos, como por exemplo, na comercialização de produtos no exterior. O dinamismo ambiental, nesta pesquisa, foi mensurado por indicadores desenvolvidos por Carvalho e Rossetto (2014), seguindo proposição conceitual elaborada por Lumpkin e Dess (2001).

\section{Capacidade Absortiva e Dinamismo Ambiental}

Gray (2006) afirma que é cada vez mais importante o papel da tecnologia da informação em compensar estas limitações no enfrentamento do dinamismo ambiental percebido, alavancando a possibilidade de absorção de conhecimento (capacidade absortiva) e a produção de conhecimento técnico específico para a atividade considerada, substituindo o papel da pesquisa e desenvolvimento necessários. A firma necessita desenvolver capacidades dinâmicas e de absorção do conhecimento em alto nível, para ir ao encontro das necessidades dos consumidores (COVIN; SLEVIN, 1989).

A capacidade de absorção de conhecimento, aliada ao aprendizado constante, é um fator crucial para redução do dinamismo ambiental e para a reação da firma às incertezas do ambiente (LIMA, 2007), tendo como resultado níveis mais altos de produtividade e desempenho (HALL, 1980). Farias (2014), afirma, nesta linha, que a absorção e transmissão de conhecimento no setor são potencializadas pela existência de várias organizações que oferecem este suporte, tais como a EMBRAPA, a APROVALE e o Instituto Federal do Rio Grande do Sul (IFRGS), entre outras, amenizando os efeitos do ambiente dinâmico.

Os conhecimentos gerados por estas instituições são compartilhados por toda a cadeia produtiva vitivinícola. Nessa perspectiva, o processo de absorção de conhecimento se realiza de diversas formas, principalmente através da interação dos agentes envolvidos. Esta interação é incrementada pela proximidade e afinidade cultural, econômica e social entre os agentes, facilitada pela linguagem similar, códigos e canais de comunicação dominados e compartilhados por todos os participantes do processo.

Li e Liu (2012), afirmam, com relação à absorção de conhecimento por parte das organizações, que o comportamento é similar à ligação existente entre os fluxos de financiamento e o dinamismo ambiental. Eles reforçam dizendo que um ambiente mais dinâmico vai requerer uma capacidade de absorção de conhecimento maior para sobrevivência da organização, e o contrário também se verifica, caracterizando uma influência moderadora ou mediadora na conexão entre dinamismo, capacidade de absorção de conhecimento (capacidade absortiva) e desempenho da firma, influência esta que é analisada neste trabalho.

Firmas operando em ambientes com alto grau de dinamismo são forçadas a desenvolver igualmente um alto grau de capacidade absortiva para minimizar o risco de obsolescência (JANSEN et al., 2006).

\section{Modelo conceitual proposto e hipóteses da pesquisa}

O modelo proposto de relacionamento das variáveis da pesquisa está exemplificado abaixo na Figura 1. A partir da apresentação do modelo conceitual, desenvolvem-se as hipóteses da pesquisa.
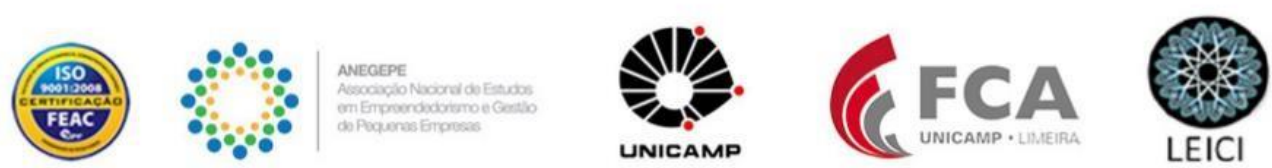
Figura 1: Modelo Conceitual

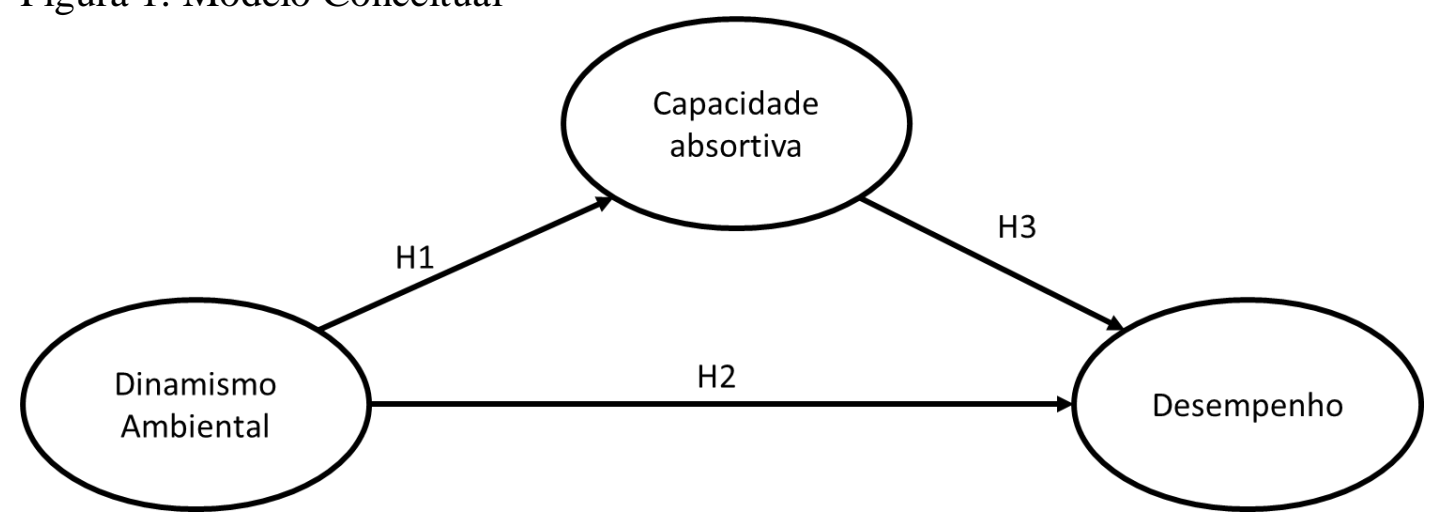

O dinamismo ambiental descreve o grau e a imprevisibilidade das mudanças no ambiente externo da organização (DESS; BEARD, 1984). Quando ele aumenta com mudanças mais rápidas e imprevisíveis em tecnologia, preferências dos consumidores, disputa por recursos e aumento da competição, a organização necessita desenvolver uma gama de capacidades para sobreviver e manter vantagem competitiva no mercado (D’AVENI, 1994). Li e Liu (2012) afirmam que ambientes caracterizados pelo aumento no dinamismo ambiental forçam as organizações a cultivar e incrementar sua capacidade absortiva. Da argumentação destes autores se propõe a hipótese 1:

\section{Hipótese 1 - $O$ Dinamismo Ambiental tem efeito positivo na Capacidade Absortiva.}

Conforme Aldrich (1979), um alto grau de dinamismo ou turbulência ambiental estimula a inovação, fazendo com que a organização tenha mais consciência dos sinais e indicações da necessidade de inovar. Estas inovações, uma vez implementadas, provocam alterações positivas em métodos e processos (FUENTES et al., 2004), criando novas capacidades dinâmicas (TEECE; PISANO; SHUEM, 1997), que resultarão em melhoria de desempenho da organização (TING, WANG, WANG, 2012). Da argumentação destes autores se propõe a hipótese 2:

\section{Hipótese 2 - $O$ dinamismo ambiental tem efeito positivo no desempenho da firma.}

A capacidade absortiva é geradora de informação para a organização (COHEN; LEVINTHAL, 1989) que, estruturada e disseminada pelas rotinas e processos, contribuirá para a melhoria do desempenho organizacional (CAMISÓN; FORÉS, 2010). Esta melhoria de desempenho impactará diretamente no ambiente operacional, ajudando a gerar vantagem competitiva, fundamental para impulsionar o seu posicionamento estratégico (ZAHRA; GEORGE, 2002), de onde se pode deduzir que o desempenho da firma tem relação direta com a sua capacidade absortiva (FLATTEN et al., 2011). Da argumentação destes autores se propõe a hipótese 3:

Hipótese 3 - A capacidade absortiva tem efeito positivo no desempenho da firma.
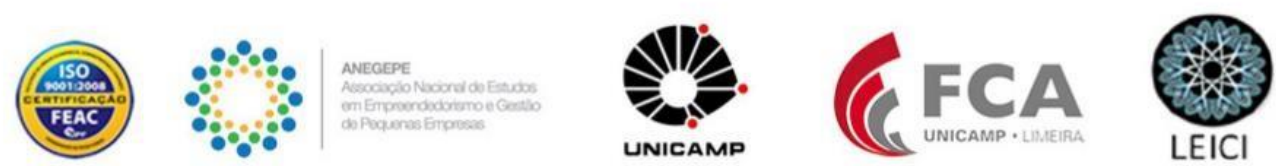


\section{Procedimentos Metodológicos}

Esta pesquisa caracteriza-se com abordagem quantitativa, de caráter descritivo, utilizando-se a técnica Survey com corte transversal (MALHOTRA, 2005). O universo considerado para a pesquisa são os produtores de vinhos finos das regiões fabricantes dos Estados de Santa Catarina, ACAVITIS (Associação Catarinense Vinhos de Altitude) de São Joaquim (SC) e Rio Grande do Sul, IBRAVIN (Instituto Brasileiro do Vinho), de Bento Gonçalves (RS), que compõe uma população de aproximadamente 180 a 200 produtores nos dois Estados, a grande maioria das empresas de base familiar. A amostra utilizada foi de 77 empresas, sendo 71 do Rio Grande do Sul, e 6 de Santa Catarina, apresentando uma idade media de 30 anos.

A construção do instrumento de coleta de dados (questionário) seguiu alguns procedimentos: primeiramente foram apresentados os indicadores ou variáveis que fizeram a mensuração de cada construto envolvido no modelo conceitual proposto, o resultado do pré-teste (pesquisadores, especialistas e produtores e comercializadores de vinho), realizado para refinamento e aprimoramento das questões do questionário e, finalmente, o procedimento a ser utilizado para coleta dos dados.

O questionário básico utilizado para esta pesquisa, no que se refere à Capacidade Absortiva, baseia-se nos formulários validados e testados por Flatten et al. (2011), Barrionuevo (2009) e Camisón e Forés (2010). O dinamismo ambiental foi mensurado por indicadores desenvolvidos por Carvalho e Rossetto (2014), seguindo proposição conceitual elaborada por Lumpkin e Dess (2001). As questões do formulário tem como opção de resposta a Escala do Tipo Likert de cinco pontos, variando de 1 (discordo totalmente) a 5 (concordo plenamente). Para mensuração do desempenho foram utilizados: "Retenção de Clientes", "Receita" e "Quantidade de Litros Produzidos", foi igualmente utilizada escala de cinco pontos, variando desde 1 (muito pior) até 5 (muito melhor).

A análise dos dados com o teste das hipóteses foi realizada por meio da Modelagem de Equações Estruturais, com a técnica Partial Least Squares, sendo utilizado o software SmartPLS. A análise pelo PLS pressupõe duas etapas distintas de análise, uma relacionada ao modelo de mensuração que avalia se os indicadores observáveis refletem adequadamente os construtos do modelo, e a última etapa que avalia os relacionamentos entre os construtos (HAIR, etal., 2014). Na Figura 2 apresentamos os indicadores de ajuste utilizados, baseados em Hair et al. (2014).

Figura 2. Indicadores de ajuste utilizados na modelagem em PLS.

\begin{tabular}{|c|c|c|c|}
\hline INDICADOR & COEF. & ITEM & VALOR ESPERADO \\
\hline Variância média extraída & Ave & Correlação entre indicadores e construtos & Acima de 0,5 \\
\hline Alpha de cronbach & Ac & Consistência da escala & Acima de 0,7 \\
\hline Confiabilidade Composta & $\mathrm{Cc}$ & Consistência da escala & Acima de 0,6 \\
\hline Validade discriminante & $\mathrm{Vd}$ & Unidimensionalidade & Maior carga Fatorial \\
\hline Fator de aumento da Variância & Vif & Colinearidade dos indicadores & Abaixo de 3,3 \\
\hline
\end{tabular}
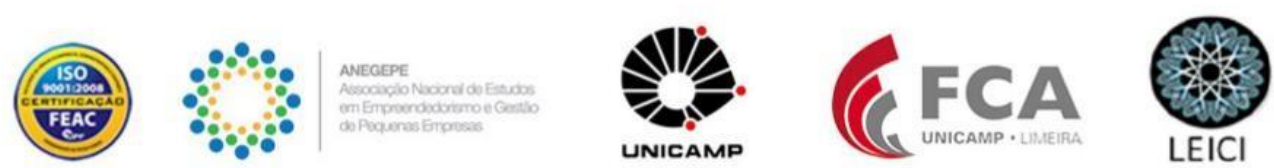


\begin{tabular}{|c|c|c|c|}
\hline INDICADOR & COEF. & ITEM & VALOR ESPERADO \\
\hline Validade preditiva & $\mathrm{Vp}$ & Colinearidade dos construtos & Abaixo de 3,3 \\
\hline Variância explicada & $\mathrm{R}^{2}$ & Capacidade de explicação do modelo estrutural & $\begin{array}{l}\text { - de } 0,02 \text { a } 0,13 \text { - fraco } \\
\text { - de } 0,13 \text { a } 0,26 \text { - médio } \\
>0,26 \text { - forte }\end{array}$ \\
\hline Poder de relacionamento das variáveis & $\mathrm{F}^{2}$ & Relevância dos construtos para o ajuste do modelo & $\begin{array}{l}\text { - de } 0,02 \text { a } 0,15 \text { - fraco } \\
\text { - de } 0,15 \text { a } 0,35 \text { - médio } \\
>0,35 \text { - forte }\end{array}$ \\
\hline Poder de ajuste do modelo & $\mathrm{Q}^{2}$ & $\begin{array}{l}\text { Expectativa de ajuste do modelo ajustado em relação ao } \\
\text { esperado redundâncias }\end{array}$ & $\begin{array}{l}\text { Valores maiores do que } \\
\text { zero }\end{array}$ \\
\hline Coeficientes de caminho & $\mathrm{T}$ & Significância das relações entre os construtos & $\begin{array}{l}\text { Valores mínimos } \\
\text { - alpha } 5 \%-1,96 \\
\text { - alpha } 1 \%-2,58 \\
\text { - alpha } 0,1 \%-3,29\end{array}$ \\
\hline
\end{tabular}

\section{Resultados da Pesquisa}

O modelo de mensuração foi avaliado utilizando-se das medidas de Variância Média Extraída - AVE, confiabilidade, confiabilidade composta, e validade discriminante. Os valores de AVE foram de 0,395 para Capacidade Absortiva, 0,522 para dinamismo e 0,619 para desempenho, sendo possível concluir que as variáveis Desempenho e Dinamismo apresentam valores acima dos mínimos requeridos para validade convergente do modelo, enquanto que Capacidade Absortiva mostra índices um pouco abaixo, mas dentro do critério de Bollen (1989) e Ping (2007), para os quais valores em torno de 0,4 são aceitáveis.

Valores de Alfa de Cronbach, utilizados para mensurar a confiabilidade das escalas ficaram em 0,728 para capacidade absortiva, 0,844 para desempenho e 0,536 para dinamismo, sendo que esta última apresentou valores um pouco abaixo do que se esperava. Valores de confiabilidade composta ficaram em 0,805 para capacidade absortiva, 0,889 para desempenho e 0,764 para dinamismo. A validade discriminante foi testada pelo critério de cargas cruzadas e pelo critério de Fornell e Lacker (1981), tendo sido aprovada em ambos. Assim, embora tenhamos tido valores um pouco abaixo de AVE para capacidade absortiva, e de Alfa de Cronbach para dinamismo, os demais indicadores de qualidade de mensuração, todos ficaram acima dos valores esperados, sendo assim considerado validado o modelo de mensuração.

A análise do modelo estrutural foi realizada por meio da avaliação, do coeficiente de determinação $-\mathrm{R}^{2}$, da análise do poder de relacionamento entre as variáveis do modelo pelos indicadores de Cohen - $\mathrm{f}^{2}$ e de Stone-Geisser - $\mathrm{q}^{2}$, e pela análise de significância dos coeficientes de caminho realizada por meio do teste $t$ de Student. O coeficiente de determinação dos dois construtos endógenos do modelo, teve os resultados de $\mathrm{R}^{2}=0,149$ para capacidade absortiva,
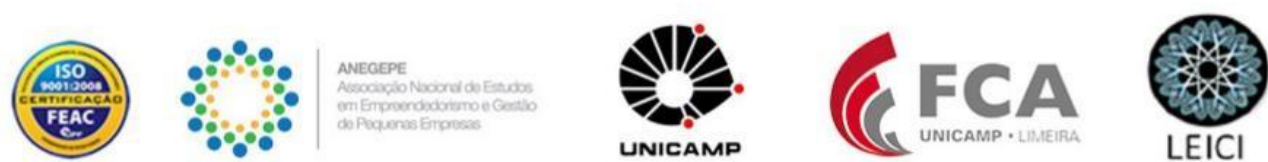
indicando que aproximadamente $15 \%$ da variância deste construto é determinada pelo construto dinamismo, e $\mathrm{R}^{2}=0,244$ para desempenho, indicando que aproximadamente $24 \%$ da variância do desempenho é determinada pelos construtos capacidade absortiva e dinamismo.

Para analisar o poder dos relacionamentos, primeiro avaliamos o indicador de Cohen (1988), que propõe que efeitos entre 0,02 e 0,15 mostram relacionamento fraco, efeitos entre 0,15 e 0,35 um efeito moderado, e acima de 0,35 , efeito forte e consistente de relacionamento entre as variáveis. Na pesquisa, o relacionamento entre capacidade absortiva e desempenho foi de 0,250 , caracterizado como moderado, o relacionamento entre dinamismo e capacidade absortiva foi de 0,165 , também caracterizado como moderado, enquanto o relacionamento entre dinamismo e desempenho ficou em 0,006, sendo considerado inexistente. Para analisar o poder de ajuste, medido pelas redundâncias, calculo do indicador de Stone-Geisser - $\mathrm{Q}^{2}$ para os construtos endógenos apresentou valores acima de zero, como esperado, sendo de 0,026 para capacidade absortiva e de 0,133 para desempenho.

Para analisar a significância dos relacionamentos entre os construtos, e enfim testar as hipóteses da pesquisa, foi realizada análise por meio do teste $t$ de Student. No software SmartPLS, este teste é realizado por meio de uma rotina de bootstrapping. Testando a hipótese 1, o efeito de dinamismo ambiental sobre a capacidade absortiva apresentou um coeficiente de caminho no valor de 0,376 ( $t$ test de 3,219, $\mathrm{p}<0,01$ ), suportando a hipótese de que quando se aumenta o dinamismo ambiental, a empresa desenvolve maior capacidade de absorver conhecimentos do ambiente.

Já a relação entre dinamismo e desempenho, prevista na hipótese 2, apresentou um valor de coeficiente de caminho de 0,073 ( $t$ testde 0,508, p > 0,05), rejeitando assim a hipótese, ou seja, o dinamismo não gera efeitos diretos sobre o desempenho.

Apresentando um valor de coeficiente de caminho de 0,468 ( $t$ test de 4,411, p < 0,001), a hipótese 3 da relação entre capacidade absortiva e desempenho foi suportada, ou seja, quanto maior a capacidade que a empresa tem de absorver conhecimentos de seu ambiente, maior será o seu desempenho. A Figura 3 apresenta o modelo conceitual após os testes de hipóteses.

Figura 3: Modelo final com testes de hipóteses.
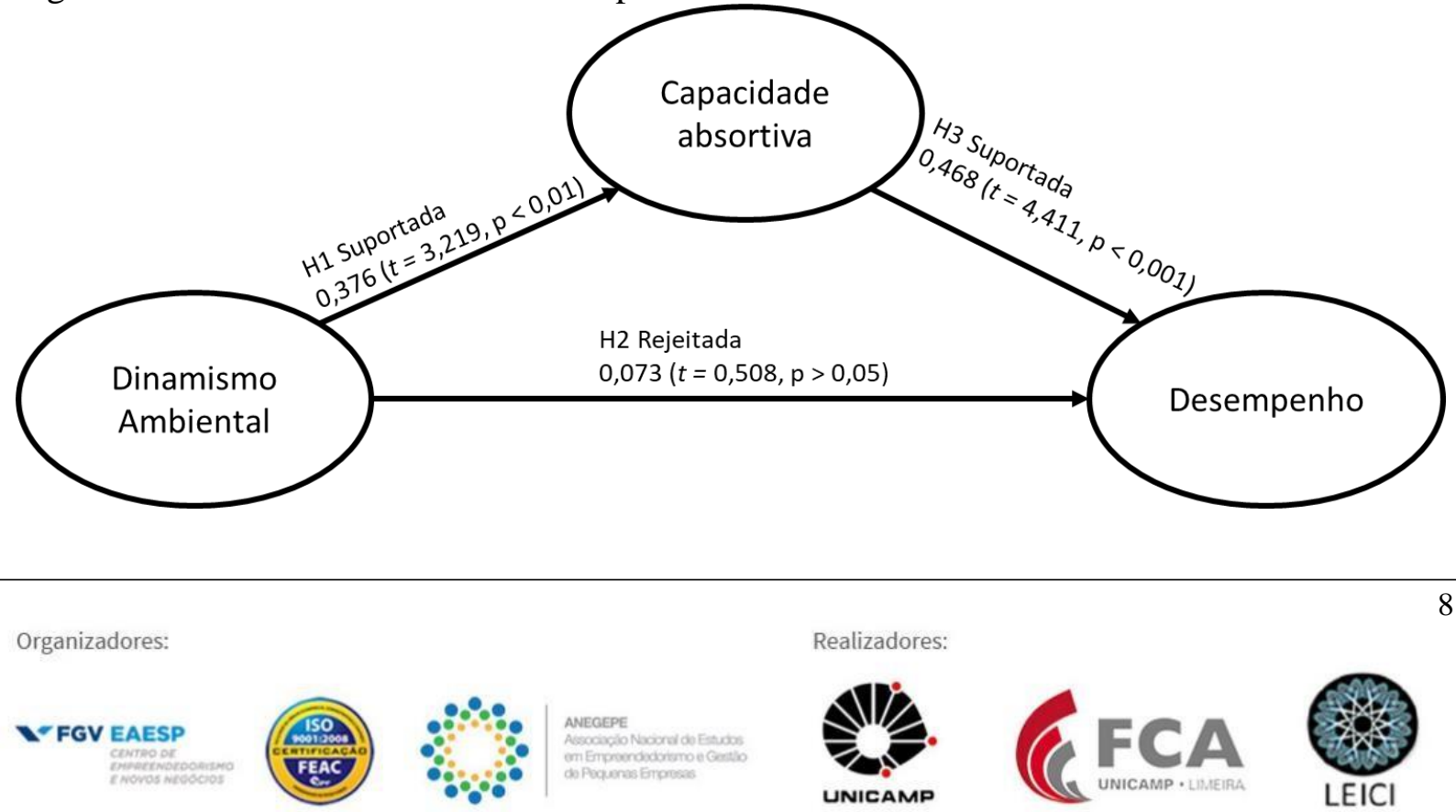

Realizadores:
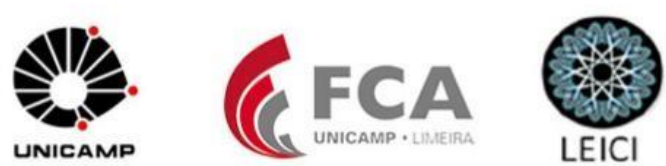


\section{Discussão dos resultados}

A aplicação da Teoria da Capacidade Absortiva apresentada por Zahra e George (2002), do diamsmo ambiental e do desempenho no universo das pequenas e médias empresas da área vitivinícola do Sul do Brasil (RS e SC), se reveste de especial importância no sentido de investigar os resultados da pesquisa em um ambiente com características consideravelmente diferentes das aplicações usuais desta teoria em suas origens, ou seja, em mercados desenvolvidos ou em universos de corporações industriais com níveis de sofisticação administrativa ou operacional bem mais elevados do que os apresentados pelas empresas que são foco deste trabalho.

Desta forma, a discussão dos resultados se deu a partir das hipóteses apresentadas no item 5 (modelo conceitual proposto e hipóteses da pesquisa) e no item 7 (resultados da pesquisa).

\section{Hipótese 1 - O Dinamismo Ambiental tem efeito positivo na Capacidade Absortiva (Suportada)}

O dinamismo ambiental descreve a taxa de mudança, imprevisibilidade, volatilidade e instabilidade no ambiente externo (Hoskisson et al., 2000; Jansen et al., 2006). Sirmon et al. (2007: 275) afirmam que o dinamismo ambiental resulta em uma grande quantidade de incerteza que "produz deficiências nas informações necessárias para identificar e entender as relações de causa e efeito".

Quando os ambientes são altamente dinâmicos, a incerteza pode inibir a capacidade de uma organização responder a necessidade de mudança, prever as demandas dos clientes, questionar a direção estratégica existente e explorar novas alternativas (González-Benito et al. 2012). No entanto, um ambiente turbulento em que as mudanças externas não são lineares e descontínuas também pode ser uma ótima fonte de oportunidades para que as empresas fortaleçam suas capacidades existentes e/ou desenvolvam novas que permitam que as empresas superem sua inércia organizacional e miopia de aprendizagem (Schilke, 2014).

Em consonância com isso, Eisenhardt e Martin (2000) afirmam da necessidade de uma forte de inteligência de mercado, conhecimento rápido e aplicação desse conhecimento ao produto e às decisões de mercado para que as empresas possam abordar mudanças em ambientes dinâmicos. Zahra et al. (2006) sugerem que os ambientes de alta incerteza compelem as empresas a desenvolver melhor gestão do conhecimento que promovam a criação de novos conhecimentos específicos da situação e melhorem o pensamento criativo que levam a um desempenho de inovação superior.

Se um ambiente for percebido como dinâmico, as empresas precisam usar os repositórios de conhecimento existentes mais eficazes e melhorar a sua capacidade absortiva através de informações em tempo real, redes interativas e comunicação intensiva, apoiada pelas habilidades de TI para ajudar a unir as experiências de aprendizagem não lineares umas às outras e aos resultados da inovação (Eisenhardt 1989; Yayavaram e Chen 2015).

A pesquisa de Liu et al. (2013) mostra que as empresas do mercado emergente que operam em ambientes de negócios dinâmicos adquiriram diferentes capacidades de gerenciamento de
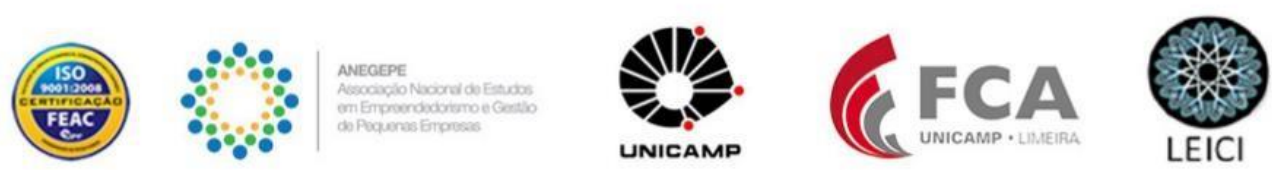
conhecimento e aprendizagem em comparação com empresas em países desenvolvidos. Assim, no contexto de um alto dinamismo, as empresas com capacidade absortiva mais fortes fazem melhor sentido de ambientes ambíguos e, portanto, são capazes de tomar o curso certo de ações resultantes de um melhor desempenho.

\section{Hipótese 2 - $O$ dinamismo ambiental tem efeito positivo no desempenho da firma (Rejeitada)}

A não validação desta hipótese pode ser explicada buscando argumento em diversos pesquisadores organizacionais de diversas disciplinas de pesquisa quando afirmam que no ambiente dinâmico de hoje, as organizações precisam ser ambidestras - alinhadas e eficientes no gerenciamento das demandas comerciais atuais, ao mesmo tempo que são adequadas o suficiente para atender às demandas do meio ambiente que provavelmente irão encontrar amanhã (Helfat et al.2007; Jansen, Van Den Bosch e Volberda, 2005; Sirmon, Hitt e Irlanda 2007; Teece, 2007).

O que parece estar nos dizendo a não validação da hipótese é que em ambientes dinâmicos, a busca do desempenho ocorrera pelo aprofundamento de uma posição estratégica, por exemplo, à medida que as complementaridades entre as atividades crescem com aprendizagem acumulada e capacidades que ocorrem à medida que as atividades são realizadas. Nesta perspectiva, o que se afirma é a necessitada das organizações estudadas desenvolverem capacidades dinâmicas (capacidade de absorção do conhecimento) como um meio de, em ambiente dinâmicos, buscar desempenho. A capacidade dinâmica serviria como um mediador desta relação.

Os avanços recentes na literatura de capacidades dinâmicas identificam a capacidade de uma empresa de alcançar "orquestração de recursos de melhoramento de valor" como uma das micro bases das capacidades dinâmicas das empresas para gerar desempenho (Teece 2007, p. 1344). De acordo com essa perspectiva, as empresas, em ambientes dinâmicos, criam valor de longo prazo através de atividades de detecção, apreensão e transformações que ajudam as empresas a combinar e reconfigurar ativos especializados para atender às demandas do mercado em mudança (Teece 2007).

Ainda com relação ao dinamismo ambiental, é interessante perceber que os resultados desta pesquisa coincidem com os encontrados por Carvalho (2011), em um estudo em hotéis em nível de Brasil, mas com predominância da região Sul (PR, SC e RS), o qual afirma que o dinamismo não apresenta efeitos diretos sobre o desempenho, apenas indiretos, através da orientação empreendedora, concluindo que as organizações poderão elevar seu padrão de desempenho quando o dinamismo as conduzir a adotar estratégias empreendedoras.

Pode-se observar nos resultados a minimização dos efeitos do dinamismo ambiental, no caso específico das PME's da área vitivinícola, pela ação das associações e cooperativas e pela atuação das empresas estudadas na forma de redes de cooperação, fazendo com que o dinamismo ambiental não apresente relação com o desempenho.

Uma das razões aferidas para a não validação desta hipótese é o efeito de proteção oferecido pelas redes de cooperação no caso das PME's estudadas, conforme Lundvel (1988), Maskell e Malmberg (1999) e Farias (2014). Esta proteção é resultante dos efeitos da proximidade e

Organizadores:

- fGV EAesp

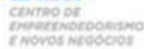
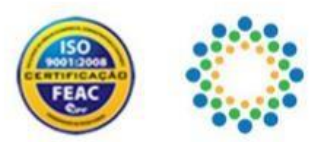

ANEGEPE

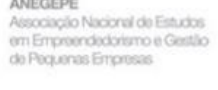

Realizadores:

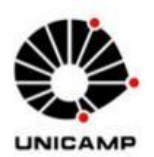

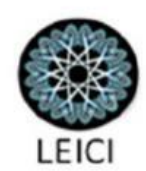


afinidade cultural e econômica e do compartilhamento do conhecimento e experiências que geram aprendizado coletivo entre as empresas. Esta situação tem efeito importante nas práticas organizacionais, pois a proteção e as informações geradas pela rede de relacionamentos favorece e facilita a tomada de decisões gerenciais, o que de outra forma poderia ter resultados desastrosos para as empresas.

Reforçando, observa-se também que o dinamismo é percebido de forma diferenciada pelas pequenas e médias empresas (TEIXEIRA; ROSSETTO; CARVALHO, 2009), devido, em parte, às suas limitadas bases de recursos e à alta concentração de decisões gerenciais nas mãos de poucas pessoas ou de um único gestor. Isto fica bem claro nos resultados do estudo, que conclui que o dinamismo ambiental não tem influência no desempenho das empresas estudadas.

\section{Hipótese 3 - A capacidade absortiva tem efeito positivo no desempenho da firma (Suportada)}

Com relação à capacidade absortiva, a presente pesquisa demonstra o seu efeito positivo no desempenho das PME's da área vitivinícola, cuja explicação reside justamente na ação do fornecimento de conhecimento através da rede de relacionamentos e, associações, cooperativas, independentemente do tamanho ou sofisticação organizacional e administrativa da firma, gerando possibilidade de desempenho igualitário entre todos os integrantes da rede.

Toda dimensão do processo de absorção do conhecimento é, em certa medida, relevante para o desempenho, uma vez que as quatro etapas são importantes para o desenvolvimento efetivo do processo de absorção do conhecimento como um todo (Todorova e Durisin, 2007)

Hood (2007) afirma que a falta de investimentos em processos de conhecimento provavelmente reduz a capacidade da empresa de alcançar desempenho. Nas empresas pesquisadas, o investimento em capacidade de absorção aumentará ao longo do tempo sua vantagem relativa de desempenho em relação àquelas que não conseguem investir. Meyer e Zucker (1989) afirmam baixos investimentos em abosorção de conhecimento deixarão as empresas para trás, sendo cada vez mais difícil recuperar o terreno e, em última instância, podem se tornar organizações permanentemente incapazes de alcançar desempenho.

O principal valor da comprovação desta hipotese é que fornece uma maneira de formular uma compreensão do desempenho das organizações do setor vinicolca do sul do Brasil em termos de processos de conhecimento. As teorias do gerenciamento do conhecimento destacam o requisito de realizar organizações com êxito para criar novos conhecimentos relacionados a tarefas, disseminálo em toda a organização e incorporá-lo em seus produtos, serviços e sistemas (Marshall et al., 1996).

As literaturas sobre capacidade de absorção, capacidades dinâmicas, aprendizagem organizacional e gestão do conhecimento desenvolveram-se, em grande medida, independentemente uma das outras. Somente agora links começam a ser feitos, pois os estudiosos preocupados com a explicação do desempenho organizacional identificaram os benefícios potenciais de uma convergência entre essas abordagens (Easterby-Smith e Prieto, 2008).

Nossa análise mostra que a capacidade de absorção pode complementar e ajudar a integrar algumas dessas teorias existentes de gerenciamento de conhecimento e processamento de
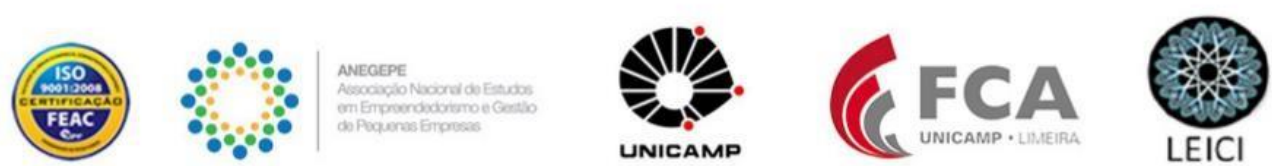
conhecimento em relação ao desempenho organizacional. Por exemplo, o modelo de Lane et al. (2006) encapsula uma ampla gama de variáveis que atuam como mediadores entre conhecimento e desempenho organizacional subseqüente, enquanto Cohen e Levinthal (1990) e Van den Bosch et al. (2003) propõem uma série de relações teóricas que examinam processos específicos de aprendizagem em relação à capacidade de absorção. Easterby-Smith e Prieto (2008) estabeleceram uma estrutura que integra essas duas abordagens e oferece um caminho a seguir para a pesquisa. Isso mostra que a capacidade de absorção pode fornecer uma maneira útil de entender a maneira como os processos de aprendizagem e a gestão do conhecimento influenciam o desempenho organizacional.

No geral, nossa análise mostra que a aplicação da teoria da capacidade de absorção ao setor vinicola tem uma alta relevância. A análise se baseou nas dimensões de aquisição, assimilação, transformação e aplicação de conhecimento e oferece um meio de avaliação ex ante que pode facilitar a ação no alcance do desempenho.

Ao avançar nesta agenda de pesquisa, os teóricos que buscam estudar a capacidade de absorção como uma teoria antecessora na análise do desempenho precisarão abordar uma série de desafios. Estes incluem a realização de pesquisas que ajudem a desenvolver a teoria sobre como os processos de conhecimento operam dentro das organizações, construindo medidas e conjuntos de dados que reflitam esses processos de conhecimento, considerando gerentes envolvidos na gestão e melhoria do desempenho e pensando em capacidade de absorção em relação à gestão colaborativa e ao trabalho multiorganizacional.

\section{Conclusões}

Neste trabalho procurou-se analisar a pequena e média empresa vitivinícola do Sul do Brasil sob a ótica capacidade absortiva, dinamismo ambiental e o efeito destas no desempenho destas nas empresas. Como um dos resultados da análise, concluiu-se que o dinamismo afeta positivamente a capacidade das empresas absorverem conhecimento, isto é, em ambientes com forte grau de incerteza, para entender melhor o que acontece neles, as empresas necessitam investir em processos de absorção de conhecimento, ou seja, capacidade absortiva.

Outro resultado foi que o dinamismo não gera efeito significativo sobre o desempenho. Na prática, isto significa que, quando o gestor percebe o ambiente como dinâmico, deve adotar ferramentas de gestão para enfrentar este dinamismo. Em ambientes dinâmicos como os do setor vitivinícola, portanto, é fundamental que as empresas adotem ou assimilem posturas que lhes levem a uma maior absorção de conhecimento para minimizar os efeitos do dinamismo ambiental.

Observou-se também que a capacidade absortiva gera forte efeito no desempenho da firma, significando que, provavelmente, nas pequenas e médias empresas da área vitivinícola, a absorção de conhecimento por parte dos gestores é fundamental para o sucesso do empreendimento. $\mathrm{Na}$ prática, é interessante procurar os alicerces deste mecanismo no setor vitivinícola, construído por redes de cooperação e disseminação de conhecimento, encabeçadas principalmente pela EMBRAPA (Empresa Brasileira de Pesquisa Agropecuária.

Portanto, é perfeitamente compreensível a atuação da EMBRAPA, e também das associações

Organizadores:

- fov eaesp

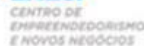
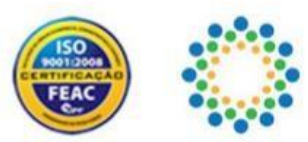

ANEOEPE

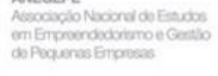
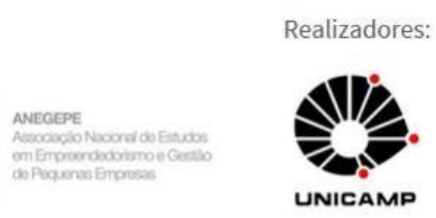
regionais (especialmente IBRAVIN e APROVALE no Rio Grande do Sul, e ACAVITIS em Santa Catarina) no fornecimento de conhecimento e capacitação gerencial que resultam em aumento da capacidade absortiva e consequente diminuição do dinamismo ambiental. No caso específico das PMEs da área vitivinícola do Sul do Brasil, o desempenho se mostra intensamente dependente da capacidade absortiva para reduzir a incerteza.

Respondendo à pergunta da pesquisa, podemos inferir que as relações extraídas dos resultados desta pesquisa entre a Capacidade Absortiva, o Dinamismo Ambiental e o Desempenho são específicas do setor da PME da indústria vitivinícola do RS e SC, assim como também se confirma o arcabouço teórico utilizado neste trabalho.

A presente pesquisa foi limitada ao Vale dos Vinhedos, na Serra Gaúcha e nas vinícolas da Serra Catarinense, e lidou somente com as vinícolas que trabalham na sua maior parte com uvas do tipo Vitis vinífera, variedades produtoras dos vinhos conhecidos como "vinhos finos", de maior valor agregado e sem função de alimentação ou produção de suco de uva.

Outra limitação da pesquisa, geradora de sugestão para futuras pesquisas, é a possibilidade de se ampliar o trabalho para países vitivinícolas como Portugal, Espanha, Itália e França, por exemplo, onde se poderiam analisar as diferenças entre os níveis de capacidade absortiva observados e seus efeitos sobre o desempenho e o dinamismo do mercado local em relação ao ambiente do Sul do Brasil. Mais ainda, sobre a economia vitivinícola do país analisado, traçando um paralelo entre diferentes culturas. Também se pode sugerir um trabalho mais abrangente, incluindo todo o setor vitivinícola, e não somente o de vinhos finos, para uma avaliação mais generalizada do efeito e da importância da capacidade de absorção de conhecimento sobre este setor da economia.

Em termos técnicos, achamos importante como sugestão de pesquisa analisar o desempenho da escala utilizada e desenvolvida para obter os resultados em outros contextos. Fica também a sugestão de uma pesquisa longitudinal, avaliando de que forma as diferentes dimensões da capacidade absortiva podem evoluir através do tempo e seu papel no desenvolvimento e na criação de novos conhecimentos com impacto relevante no crescimento das empresas. Por outro lado, seria interessante estudar quais variáveis internas das PME's são mais importantes para o desenvolvimento da capacidade absortiva e em quais dimensões da teoria de Zahra e George (2002) se encaixam melhor estas variáveis, cuja pesquisa seria de particular importância, em termos práticos, para o desenvolvimento e o aperfeiçoamento das estruturas, competências e habilidades organizacionais e gerenciais nas pequenas e médias empresas.

\section{Referências}

ALDRICH H. E. Organizations and Environments. Englewood Cliff: NJ. Prentice-Hall, 1979. ANDERSEN, M. N. Eco-Innovation Indicators. Background Paper For The Workshop On EcoInnovation Indicators. Copenhagen, Sept. 29, EEAeletronicreport, Copenhagen. 2005.

BARRIONUEVO, M. M. Tesis. Universidad Granada. Influencia de la Capacidad de Absorber Conocimiento en la Capacidad Estrategican Empreendedora: um modelo casual en Empresas Españolas, 2009.

BOLLEN, K. A. Structural Equations with Latent Variables, New York: John Wiley \& Sons,

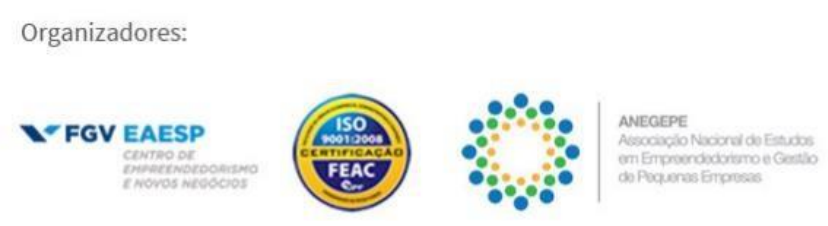

Realizadores:
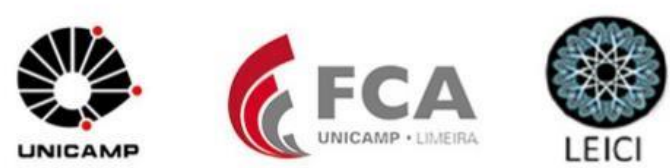
Inc, 1989.

BOSKIN, M.; STIGLITZ, J.; NISKANEN, W.; PENNER, R. Policy and Process: Where do we go from here? Contemporary Economic Policy, v. 3, pag. 53-78, 1984.

CAMISÓN, C; FORÉS, B. Knowledge Absorptive Capacity: New Insights for Its

Conceptualization and Measurement. Journal of Business Research, 63, 707-715, 2010.

CARVALHO, C. O Relacionamento Entre Ambiente Organizacional, Capacidades Estratégicas, Orientação Estratégica e Desempenho: um Estudo no Setor Hoteleiro Brasileiro. Tese de Doutorado em Administração e Turismo, 2011, UNIVALI-SC.

CARVALHO, C. E.; ROSSETTO, C. R. Proposição e teste de uma escala de dinamismo, complexidade e munificência ambiental. Revista Ibero-Americana de Estratégia, v. 13, n. 4, p. 59-73, 2014.

COHEN, J. Statistical power analysis for the behavioral sciences. Hillsdale: Erlbaum, 1988. COHEN, W; LEVINTHAL,D. Innovation and Learning: The Two Faces of R\&D. Economic Journal, 99 , 569-596, 1989.

COHEN, W, LEVINTHAL,D. Absorptive Capacity: A New Perspective on Learning and Innovation. Administrative Science Quarterly, 35, 128-152, 1990.

COVIN, J. G.; SLEVIN, D. P. Strategic Management of small firms in hostile and benign environments. Strategic Management Journal, [S. 1.], v. 10, p. 75-97, 1989.

D'AVENI, D. S. The Age of Temporary Advantage. Strategic Management Journal, p.1371$1385,2010$.

D’AVENI, R. A.. Hypercompetition: Managing the Dynamics of Strategic Manoeuvering. The Free Press, New York, 1994.

DESS, G.; BEARD, D. Dimensions of Organizational Task Environments. Administrative Science Quarterly, Vol. 29, 52-73, 1984.

EASTERBY-SMITH, M.; PRIETO, I. M. Dynamic Capabilities and Knowledge Management: An Integrative Role for Learning. British Journal of Management, 19: 3 pp. 235-49, 2008. EISENHARDT, K.M.. Making fast strategic decisions in high-velocity environments. Academy of Management Journal, 32: 543-576, 1989.

EISENHARDT, K.M.; J. Martin. Dynamic capabilities: What are they? Strategic Management Journal, 21: 1105-1121, 2000.

FARIAS, C. V. S. Inovação e ganhos competitivos na vitivinicultura gaúcha: uma abordagem preliminar. Anais... Encontro de Economia Catarinense, IV, Criciúma, 2010.

FLATTEN, T.; ENGELEN, A. ZAHRA, S.; BRETTEL, M. A Measure of Absorptive Capacity: Scale Development and Validation. European Management Journal, 29, 98-116, 2011.

FORNELL, C. AND D. LARCKER. Evaluating structural equation models with unobservable variables and measurement error. Journal of Marketing Research, 18: 39-50, 1981.

FUENTES - FUENTES, M. M., ALBACETE -SAEZ, C. A., \& LLORENS -MONTES, F. J. The impact of environmental characteristics on TQM principles and organizational performance. The International Journalof Management Science, 32(6), 425 - 442, 2004.
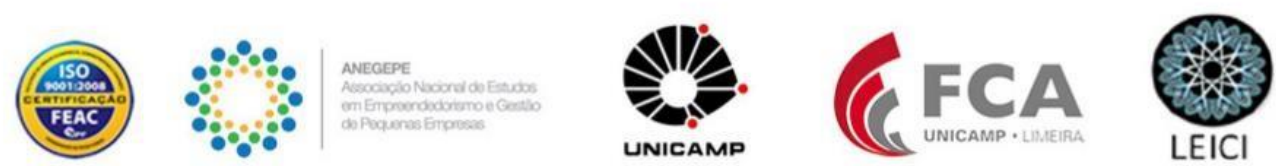
GONZÁLEZ-BENITO, J.; AGUINIS, H.; BOYD, B. K.; SUÁREZ-GONZÁLEZ, I. Coming to consensus on strategic consensus: A mediated moderation model of consensus and performance. Journal of Management, 38: 1685-1714, 2012.

GRAY, C. Absorptive Capacity, Knowledge Management and Innovation in Entrepreneurial Small Firms. International Journal of Entrepreneurial Behavior \& Research, 12(6), 345-360, 2006.

HAIR, et al. A Primer on Partial Least Squares Structural Equation Modeling (PLS-SEM). Los Angeles: SAGE, 2014.

HALL, D.J., Strategy Follows Structure. Strategic Management Journal, V.1, Issue 2, pages 149-163, April/June, 1980.

HOOD, C. Public Service Management by Numbers: Why Does It Vary? Where Has It Come from? What Are the Gaps and the Puzzles? Public Money and Management , 27: 2 pp95-102, 2007.

HSIEN C. ,CHONG Y.,. Customer needs as moving targets of product development: a review. International Journal of Advanced Manufacturing Technology, 2009.

HOSKISSON, R. E.; EDEN, L.; LAU, C.M.; WRIGHT, M. Strategies in emerging economies. Academy of Management Journal 43: 249-267, 2000.

JANSEN, J.; VAN DEN BOSCH, F.; VOLBERDA, H. Managing Potencial and Realized Absorptive Capacity: How do Organizational Antecedents Matter ? Academic of Management Journal, Vol. 48, 999-1015, 2005.

JANSEN, J.J.P., VAN DEN BOSCH, F.A.J. \& VOLBERDA, H.W. Exploratory innovation, exploitative innovation, and performance: Effects of organizational antecedents and environmental moderators. Management science, 52(11), pag.1661-1674, 2006.

JANSEN, J. J.P.; VERA, D.; CROSSAN, M.. Strategic leadership for exploration and exploitation: The moderating role of environmental dynamism. The Leadership Quarterly 20: 5-18, 2009.

LANE, P.J.; KOKA, B.; PATHAK, S. The Reification of Absorptive Capacity; A Critical Review and Rejuvenation of the Construct. Academy of Management Review, 31 (4), 833-863, 2006.

LEVIN, D.; CROSS, R. The strength of weak ties you can trust: The mediating role of trust in effective knowledge transfer. Management Science, Vol. 50, N. 11, pp. 1477-1490, 2011.

LI, D.; LIU, J. Dynamic Capabilities, Environmental Dynamism, and Competitive Advantage: Evidence From China. Journal of Business Research, Vol. 67, 2793-2799, 2011.

LIMA, G.; SAVARIZ, C.; SILVA, M. Relação entre Incerteza Ambiental e Estratégia Competitiva na Utilidade do Orçamento: Estudo em Cervejarias, UnB / FACE, 2007.

LIU, H.; JIANG, X.; Zhang, J.; ZHAO, X.. Strategic flexibility and international venturing by emerging market firms: The moderating effects of institutional and relational factors. Journal of International Marketing, 21: 79-98, 2013.

LUMPKIN, G.; DESS, G. Linking two dimensions of entrepreneurial orientation to firm performance: The moderating role of environment and industry life cycle. Journal of Business Venturing, vol. 16, issue 5, 429-45, 2001.

Organizadores:

- fov eaesp

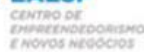
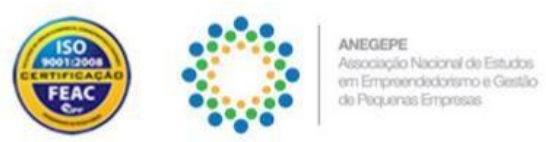

Realizadores:
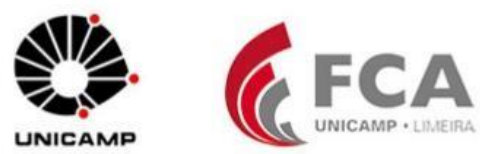
MALHOTRA, A. et al. Absorptive capacity configurations in supply chains: gearing for partnerenabled market knowledge creation. MIS Quarterly, v.29, n.1, p. 145-87, 2005.

MARSHALL, C.; PRUSAK, L.; SHPILBERG, D. Financial Risk and the Need for Superior Knowledge. Management, California Management Review, 38: 3 pp77-101, 1996.

MEYER, M. W.; ZUCKER, L. G. Permanently Failing Organizations. London: Sage. 1989.

MILES, M. P.; COVIN, J. G.; \& HEELEY, M. B. The Relationship between Environmental Dynamism and Small Firm Structure, Strategy, and Performance. Journal of Marketing Theory and Practice, 8:2, 63-78, 2015.

NDIEGE, J; HERSELMAN, M.;FLOWERDAY, S. Absorptive Capacity: Relevancy for Large and Small Enterprises, SA. Journal of Information Management, 14(1), Art 520, 2012.

PING, R. A. (2007). "Is there any way to improve Average Variance Extracted (AVE) in a Latent Variable (LV) X?" [on-line paper]. http://home.att.net/ rpingjr/LowAVE.doc.).

SCHILKE, O. On the contingent value of dynamic capabilities for competitive advantage: The nonlinear moderating effect of environmental dynamism. Strategic Management Journal, 35 : 179-203, 2014.

SIRMON, D.G.; HITT, M.A.; IRELAND, R.D. 'Managing Firm Resources in Dynamic Environments to Create Value: Looking Inside the Black Box. Academy of Management Review, 32, 1, 273-292, 2007.

TEECE, D. J. Explicating dynamic capabilities: The nature and microfoundations of (sustainable) enterprise performance. Strategic Management Journal, 28 (13), 1319-1350, 2007. TEECE, D.J.; PISANO, G; SHUEM, A. Dynamic Capabilities and Strategic. Management. Strategic Management Journal, 18(7), 509-533, 1997.

TEIXEIRA, O. R. de P.; ROSSETTO, C. R. ; CARVALHO, C. E. A relação entre oambiente organizacional e o comportamento estratégico no setor hoteleiro de Florianópolis(SC). Turismo. Visão e Ação (Itajaí), v. 11, p. 157-174, 2009.

TODOROVA, G.; DURISIN, B. Absorptive Capacity: Valuing a Reconceptualization. Academy of Management Review, 32: 774-786, 2007.

VAN DEN BOSCH, F.; VAN WIJK, R.; VOLBERDA, H. Absorptive Capacity: Antecedents, Models and Outcomes. Blackwell: Handbook of Organizational Learning \& Knowledge Management, 278-301, 2003.

YAYAVARAM, S.; CHEN,W.R.. Changes in firm knowledge couplings and firm innovation performance: The moderating role of technological complexity. Strategic Management Journal, 36: 377-396, 2015.

ZAHRA, S.; GEORGE, G. Absorptive Capacity: A Review, Reconceptualization and Extension. Academy of Management Review, Vol. 27, № 2, 2002.

ZAHRA, S.A.; SAPIENZA, H. J.; DAVIDSSON, P. Entrepreneurship and dynamic capabilities: A review, model and research agenda. Journal of Management Studies, 43: 917-955, 2006.
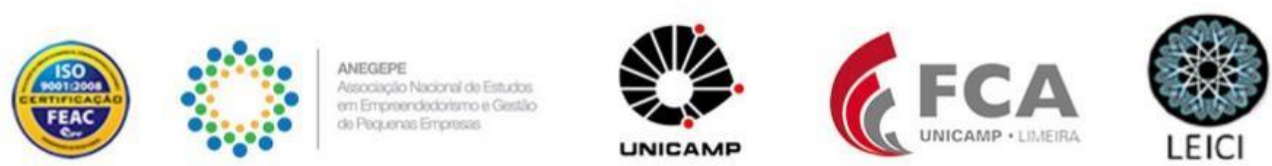